\title{
On qualitative and quantitative results for solutions to first-order dynamic equations on time scales
}

\author{
Iguer Luis Domini dos Santos ${ }^{1}$
}

Received: 11 January 2015 / Accepted: 4 May 2015 / Published online: 6 June 2015

(C) Sociedad Matemática Mexicana 2015

\begin{abstract}
In the present work, we study qualitative and quantitative results proposed in the paper Tisdell and Zaidi (Nonlinear Anal 68(11):3504-3524, 2008) of first-order dynamic equations on time scales. Thus, we examine initial value problems described by dynamic equations on time scales of the form $x^{\Delta}=f\left(t, x, x^{\sigma}\right)$. We obtain a result on the dependency of solutions to initial value problems with respect to initial values. Using Banach's fixed-point theorem, we prove the existence and uniqueness of solutions to initial value problems. On the other hand, under weaker hypothesis on $f$, using Schäfer's fixed-point theorem, we obtain the existence of at least one solution to initial value problems.
\end{abstract}

Keywords Dynamic equations - Existence of solutions - Continuous dependence · Time scales

Mathematics Subject Classification $\quad 34 \mathrm{~A} 12 \cdot 34 \mathrm{~N} 05$

\section{Introduction}

The study of first-order dynamic equations on time scales can be observed, for example, in $[1,5,6,8,10,12,15-17]$. A time scale is a nonempty closed subset of real numbers. Here, we use a bounded time scale $\mathbb{T}$, where $a=\min \mathbb{T}$ and $b=\max \mathbb{T}$ are such that $a<b$.

\footnotetext{
$凶 \quad$ Iguer Luis Domini dos Santos iguerluis@mat.feis.unesp.br

1 Departamento de Matemática, Faculdade de Engenharia de Ilha Solteira, UNESP-Univ Estadual Paulista, Rua Rio de Janeiro, 266, Ilha Solteira, São Paulo CEP 15385-000, Brazil
} 
In this study, we consider first-order dynamic equations of the type

$$
x^{\Delta}(t)=f(t, x(t), x(\sigma(t))) \quad \Delta-\text { a.e. } t \in[a, b)_{\mathbb{T}}
$$

subject to the initial condition

$$
x(a)=A
$$

where $x: \mathbb{T} \rightarrow \mathbb{R}^{n}$ is the unknown function, $A \in \mathbb{R}^{n}$ is given, $[a, b)_{\mathbb{T}}:=[a, b) \cap \mathbb{T}$, $f: \mathbb{T} \times \mathbb{R}^{n} \times \mathbb{R}^{n} \rightarrow \mathbb{R}^{n}$ is a known function, $\sigma: \mathbb{T} \rightarrow \mathbb{T}$ is a function defined in the next section, and $x^{\Delta}(t)$ is the delta derivative of $x$ at $t$.

We use the Banach fixed-point theorem to prove the existence and uniqueness of solutions to initial value problems determined by Eqs. (1) and (2). On the other hand, under weaker hypothesis on $f$, we use the Schäfer fixed-point theorem to obtain the existence of at least one solution to Eqs. (1) and (2). Furthermore, we establish a result on the dependency of solutions to Eqs. (1) and (2) with respect to initial values.

In [9], the authors consider the dynamic equation

$$
x^{\Delta}(t)=f(t, x(t), x(\sigma(t))) \quad \forall t \in \mathbb{T} .
$$

Assuming that $f$ is rd-continuous, [9] proves the existence and uniqueness of solutions to Eqs. (2) and (3). Here, we do not need to suppose that $f$ is rd-continuous to obtain the existence of solutions to Eqs. (1) and (2).

We organize the paper as follows. In Sect. 2, we recall basic concepts and results on time scale theory. Section 3 presents the results of existence and continuous dependency of solutions to first-order dynamic equations on time scales.

\section{Preliminaries}

\subsection{Time scales}

Define the forward jump operator $\sigma: \mathbb{T} \rightarrow \mathbb{T}$ by

$$
\sigma(t)=\inf \{s \in \mathbb{T}: s>t\}
$$

and the backward jump operator $\rho: \mathbb{T} \rightarrow \mathbb{T}$ by

$$
\rho(t)=\sup \{s \in \mathbb{T}: s<t\}
$$

We assume that $\inf \varnothing=\sup \mathbb{T}$ and $\sup \emptyset=\inf \mathbb{T}$.

Let $\mu: \mathbb{T} \rightarrow[0,+\infty)$ be defined by

$$
\mu(t)=\sigma(t)-t .
$$

If $A \subset \mathbb{R}$, define the set $A_{\mathbb{T}}$ as $A_{\mathbb{T}}=A \cap \mathbb{T}$. We set $\mathbb{T}^{\kappa}=\mathbb{T} \backslash(\rho(\sup \mathbb{T}) \text {, sup } \mathbb{T}]_{\mathbb{T}}$. 
Take a function $f: \mathbb{T} \rightarrow \mathbb{R}$ and $t \in \mathbb{T}^{\kappa}$. If $\xi \in \mathbb{R}$ is such that, for all $\varepsilon>0$ there exists $\delta>0$ satisfying

$$
|f(\sigma(t))-f(s)-\xi(\sigma(t)-s)| \leq \varepsilon|\sigma(t)-s|
$$

for all $s \in(t-\delta, t+\delta)_{\mathbb{T}}$, we say that $\xi$ is the delta derivative of $f$ at $t$ and we denote it by $\xi:=f^{\Delta}(t)$.

Consider a function $f: \mathbb{T} \rightarrow \mathbb{R}^{n}$ and $t \in \mathbb{T}^{\kappa}$. We say that $f$ is $\Delta$-differentiable at $t$ if each component $f_{i}: \mathbb{T} \rightarrow \mathbb{R}$ of $f$ is $\Delta$-differentiable at $t$. In this case, $f^{\Delta}(t)=\left(f_{1}^{\Delta}(t), \ldots, f_{n}^{\Delta}(t)\right)$.

The result below can be easily obtained from [2, Theorem 1.16].

Theorem 1 [2] Consider a function $f: \mathbb{T} \rightarrow \mathbb{R}^{n}$ and $t \in \mathbb{T}^{\kappa}$. Then, the following statements hold:

(i) If $f$ is $\Delta$-differentiable at then $f$ is continuous at $t$.

(ii) If $f$ is continuous at $t$ and $\sigma(t)>t$, then $f$ is $\Delta$-differentiable at $t$. Furthermore,

$$
f^{\Delta}(t)=\frac{f(\sigma(t))-f(t)}{\mu(t)} .
$$

(iii) If $\sigma(t)=t$, then $f$ is $\Delta$-differentiable at $t$ if and only if the following limit exists

$$
\lim _{s \underset{\mathbb{T}}{\rightarrow} t} \frac{f(t)-f(s)}{t-s}
$$

as an element of $\mathbb{R}^{n}$. In this case,

$$
f^{\Delta}(t)=\lim _{s \underset{\mathbb{T}}{\rightarrow} t} \frac{f(t)-f(s)}{t-s} .
$$

(iv) If $f$ is $\Delta$-differentiable at $t$, then

$$
f(\sigma(t))=f(t)+\mu(t) f^{\Delta}(t)
$$

\subsection{Lebesgue $\Delta$-integrability}

In [7], one can find the definition of a $\Delta$-measurable set of $\mathbb{T}$. We represent the family of $\Delta$-measurable sets of $\mathbb{T}$ by $\Delta$. We also recall that $\Delta$ is a $\sigma$-algebra of $\mathbb{T}$.

Let $\mu_{\Delta}: \Delta \rightarrow[0,+\infty]$ the Lebesgue $\Delta$-measure. If $E \subset \mathbb{T}$, we say that a statement $P$ holds $\Delta$-almost everywhere $(\Delta$-a.e.) on $E$, if the set $N$ given by

$$
N=\{t \in E: P \text { does not hold at } t\}
$$

is such that $\mu_{\Delta}(N)=0$. 
A function $f: \mathbb{T} \rightarrow \overline{\mathbb{R}}$ is $\Delta$-measurable if for each $r \in \mathbb{R}$ the set $\{t \in \mathbb{T}: f(t)<r\}$ is $\Delta$-measurable. If $f: \mathbb{T} \rightarrow \mathbb{R}^{n}$ we say that $f$ is $\Delta$-measurable if each component $f_{i}: \mathbb{T} \rightarrow \mathbb{R}$ of $f$ is $\Delta$-measurable.

Let $f: \mathbb{T} \rightarrow \overline{\mathbb{R}}$ and $E \in \Delta$. We indicate by

$$
\int_{E} f(s) \Delta s
$$

the Lebesgue $\Delta$-integral of $f$ over $E$. We denote the set of functions $f: \mathbb{T} \rightarrow \mathbb{R}$ which are Lebesgue $\Delta$-integrable over $E$ by $L_{1}(E)$. If $f: \mathbb{T} \rightarrow \mathbb{R}^{n}$ is $\Delta$-measurable and $E \in \Delta, f$ is Lebesgue $\Delta$-integrable over $E$ if each component $f_{i}: \mathbb{T} \rightarrow \mathbb{R}$ is Lebesgue $\Delta$-integrable over $E$. Indicate by $L_{1}\left(E, \mathbb{R}^{n}\right)$ the set of functions $f: \mathbb{T} \rightarrow \mathbb{R}^{n}$ which are Lebesgue $\Delta$-integrable over $E$. Note that, the integration of functions $f: \mathbb{T} \rightarrow \mathbb{R}^{n}$ is considered componentwise.

Let $c$ and $d$ in $\mathbb{T}$ such that $c \leq d$. If $f: \mathbb{T} \rightarrow \mathbb{R}$ is Riemann $\Delta$-integrable on $[c, d]_{\mathbb{T}}$, we indicate by

$$
\int_{c}^{d} f(s) \Delta s
$$

the Riemann $\Delta$-integral of $f$ from $c$ to $d$.

More details on integration on time scales can be found in [7].

Let $\mathcal{B}^{m}$ denote the Borel $\sigma$-algebra of $\mathbb{R}^{m}$ and $\Delta \times \mathcal{B}^{m}$ denote the product $\sigma$-algebra between $\Delta$ and $\mathcal{B}^{m}$. By definition, $\Delta \times \mathcal{B}^{m}$ is the least $\sigma$-algebra of $\mathbb{T} \times \mathbb{R}^{m}$ that contains all products $A \times B$, where $A \in \Delta$ and $B \in \mathcal{B}^{m}$.

Proposition 1 [13] Let $f: \mathbb{T} \times \mathbb{R}^{m} \rightarrow \mathbb{R}^{n}$ be a $\Delta \times \mathcal{B}^{m}$-measurable function and $u: \mathbb{T} \rightarrow \mathbb{R}^{m}$ a $\Delta$-measurable function. Then, $g: \mathbb{T} \rightarrow \mathbb{R}^{n}$, defined by

$$
g(t)=f(t, u(t))
$$

is a $\Delta$-measurable function.

From [7], the function $\sigma: \mathbb{T} \rightarrow \mathbb{T}$ is rd-continuous. As each rd-continuous function is $\Delta$-measurable, we obtain the following consequence of the previous proposition.

Lemma 1 Assume that $f: \mathbb{T} \times \mathbb{R}^{n} \times \mathbb{R}^{n} \rightarrow \mathbb{R}^{n}$ is a $\Delta \times \mathcal{B}^{2 n}$-measurable function and $x: \mathbb{T} \rightarrow \mathbb{R}^{n}$ is a continuous function. Then, the function

$$
t \mapsto f(t, x(t), x(\sigma(t)))
$$

is $\Delta$-measurable.

\subsection{Absolutely continuous functions on time scales}

First, we note that the Euclidean norm on $\mathbb{R}^{n}$ will be denoted by $\|\cdot\|$. 
A function $f: \mathbb{T} \rightarrow \mathbb{R}^{n}$ is absolutely continuous if given $\varepsilon>0$ there exists $\delta>0$ such that

$$
\sum_{i=1}^{N}\left\|f\left(b_{i}\right)-f\left(a_{i}\right)\right\|<\varepsilon
$$

whenever $a_{i} \leq b_{i}$ and $\left\{\left[a_{i}, b_{i}\right)_{\mathbb{T}}\right\}_{i=1}^{N}$ are disjoint intervals satisfying

$$
\sum_{i=1}^{N}\left(b_{i}-a_{i}\right)<\delta
$$

Theorem 2 given below can be easily obtained from [3, Theorem 4.1].

Theorem 2 A function $f: \mathbb{T} \rightarrow \mathbb{R}^{n}$ is absolutely continuous if and only if the following assertions are valid:

1. $f$ is $\Delta$-differentiable $\Delta$-a.e. on $[a, b)_{\mathbb{T}}$ and $f^{\Delta} \in L_{1}\left([a, b)_{\mathbb{T}}, \mathbb{R}^{n}\right)$;

2. for each $t \in \mathbb{T}$ we have

$$
f(t)=f(a)+\int_{[a, t)_{\mathbb{T}}} f^{\Delta}(s) \Delta s .
$$

A function $f: \mathbb{T} \rightarrow \mathbb{R}^{n}$ is said to be an arc if it is absolutely continuous.

Proposition 2 [12] Let $f: \mathbb{T} \rightarrow[0,+\infty)$ be a function in $L_{1}\left([a, b)_{\mathbb{T}}\right)$. Given $\varepsilon>0$ there exists $\delta>0$ such that, if $A \in \Delta$ and $\mu_{\Delta}(A)<\delta$ then

$$
\int_{A} f(s) \Delta s<\varepsilon
$$

If $g \in L_{1}\left([a, b)_{\mathbb{T}}, \mathbb{R}^{n}\right)$, from the previous proposition the function $z: \mathbb{T} \rightarrow \mathbb{R}^{n}$ given by

$$
z(t)=\int_{[a, t)_{\mathbb{T}}} g(s) \Delta s
$$

is an arc.

Proposition 3 [12] Let $g \in L_{1}\left([a, b)_{\mathbb{T}}\right)$. Suppose that

$$
\int_{[c, d)_{\mathbb{T}}} g(s) \Delta s \geq 0
$$

for each $c, d \in \mathbb{T}$ such that $c<d$. Then, $g(t) \geq 0 \Delta$-a.e. $t \in[a, b)_{\mathbb{T}}$. 
Corollary 1 Consider $g \in L_{1}\left([a, b)_{\mathbb{T}}\right)$ satisfying

$$
\int_{[c, d)_{\mathbb{T}}} g(s) \Delta s=0
$$

for each $c, d \in \mathbb{T}$ such that $c<d$. Then, $g(t)=0 \Delta$-a.e. $t \in[a, b)_{\mathbb{T}}$.

Corollary 2 Let $v \in L_{1}\left([a, b)_{\mathbb{T}}, \mathbb{R}^{n}\right)$ be such that

$$
\int_{[a, t)_{\mathbb{T}}} v(s) \Delta s=0
$$

for all $t \in \mathbb{T}$. Then, $v(t)=0 \Delta$-a.e. $t \in[a, b)_{\mathbb{T}}$.

\section{Main results}

Here, we obtain the existence of solutions to Eqs. (1) and (2) and the continuous dependency of solutions to Eqs. (1) and (2) with respect to initial values.

\subsection{Continuous dependency of solutions to Eqs. (1) and (2)}

We say that a function $f: \mathbb{T} \rightarrow \mathbb{R}$ is rd-continuous when it is continuous at every right-dense point $t \in \mathbb{T}$ and its left-sided limits exist and is finite at every left-dense point $t \in \mathbb{T}$. We denote the set of all rd-continuous functions with domain $\mathbb{T}$ and taking values on $\mathbb{R}$ by $C_{r d}(\mathbb{T}, \mathbb{R})$.

The set of regressive functions, $\mathcal{R}$, is defined by

$$
\mathcal{R}:=\left\{p \in C_{r d}(\mathbb{T}, \mathbb{R}): 1+\mu(t) p(t) \neq 0, \forall t \in \mathbb{T}\right\}
$$

and the set of positively regressive functions, $\mathcal{R}^{+}$, by

$$
\mathcal{R}^{+}:=\left\{p \in C_{r d}(\mathbb{T}, \mathbb{R}): 1+\mu(t) p(t)>0, \forall t \in \mathbb{T}\right\}
$$

If $p \in \mathcal{R}$, we define the exponential function $e_{p}(t, a)$ on the time scale $\mathbb{T}$ as

$$
e_{p}(t, a)=\exp \left(\int_{a}^{t} \xi_{\mu(\tau)}(p(\tau)) \Delta \tau\right)
$$

with

$$
\xi_{h}(z)= \begin{cases}\frac{\log (1+\mathrm{hz})}{h} & \text { if } h \neq 0 \\ z & \text { if } h=0\end{cases}
$$


and $\log$ is the principal logarithm function. The exponential function $e_{p}(t, a)$ is the unique solution to the initial value problem

$$
x^{\Delta}=p(t) x, \quad x(a)=1 .
$$

The next lemma, known as Gronwall's lemma on time scales, will be used in the Theorems 3 and 7.

Lemma 2 [2] Consider the functions $y \in C_{r d}(\mathbb{T}, \mathbb{R})$ and $p \in \mathcal{R}^{+}$, with $p \geq 0$ on $\mathbb{T}$. If $\alpha$ is a constant and

$$
y(t) \leq \alpha+\int_{a}^{t} p(s) y(s) \Delta s
$$

for all $t \in \mathbb{T}$, then

$$
y(t) \leq \alpha e_{p}(t, a)
$$

for all $t \in \mathbb{T}$.

A solution to Eqs. (1) and (2) is an arc $x: \mathbb{T} \rightarrow \mathbb{R}^{n}$ that satisfies the Eqs. (1) and (2).

In the next lemma, we have a result concerning the solutions of the Eqs. (1) and (2).

Lemma 3 Let $f: \mathbb{T} \times \mathbb{R}^{n} \times \mathbb{R}^{n} \rightarrow \mathbb{R}^{n}$ be a $\Delta \times \mathcal{B}^{2 n}$-measurable function.

1. If an arc $x: \mathbb{T} \rightarrow \mathbb{R}^{n}$ is a solution of the Eqs. (1) and (2) it follows that

$$
x(t)=A+\int_{[a, t)_{\mathbb{T}}} f(s, x(s), x(\sigma(s))) \Delta s \quad \forall t \in \mathbb{T} .
$$

2. If an arc $x: \mathbb{T} \rightarrow \mathbb{R}^{n}$ satisfies the Eq. (4), then $x$ is a solution of Eqs. (1) and (2).

Proof The proof is an easy consequence of the Corollary 2 and Theorem 2.

In the next theorem, we consider the following first-order dynamic equation

$$
y^{\Delta}(t)=f\left(t, y(t), y(\sigma(t)) \quad \Delta-a . e . \quad t \in[a, b)_{\mathbb{T}}\right.
$$

subject to the initial condition

$$
y(a)=B
$$

where $B \in \mathbb{R}^{n}$.

Below we state and prove the result on the dependency of solutions to Eqs. (1) and (2) with respect to initial values.

Theorem 3 Consider a $\Delta \times \mathcal{B}^{2 n}$-measurable function $f: \mathbb{T} \times \mathbb{R}^{n} \times \mathbb{R}^{n} \rightarrow \mathbb{R}^{n}$. Suppose that there exists $L>0$ such that

$$
\left\|f\left(t, p_{1}, p_{2}\right)-f\left(t, q_{1}, q_{2}\right)\right\| \leq L\left(\left\|p_{1}-q_{1}\right\|+\left\|p_{2}-q_{2}\right\|\right)
$$


for any $t \in \mathbb{T}$, and any $p_{1}, p_{2}, q_{1}, q_{2} \in \mathbb{R}^{n}$. Let $x(t)$ be a solution of the Eqs. (1) and (2) and let $y(t)$ be a solution of the Eqs. (5) and (6). If $\delta=\|A-B\|$ and $L(b-a)<1$, then for all $t \in \mathbb{T}$ we have

$$
\|x(t)-y(t)\| \leq 2 \delta M e_{2 L M}(t, a)
$$

where $M=\frac{1}{1-(b-a) L}$.

Proof For each $t \in \mathbb{T}$ we have

$$
\begin{aligned}
& \|x(t)-y(t)\|+\|x(\sigma(t))-y(\sigma(t))\| \\
& \leq 2 \delta+\int_{[a, t)_{\mathbb{T}}}\|f(s, x(s), x(\sigma(s)))-f(s, y(s), y(\sigma(s)))\| \Delta s \\
& +\int_{[a, \sigma(t))_{\mathbb{T}}}\|f(s, x(s), x(\sigma(s)))-f(s, y(s), y(\sigma(s)))\| \Delta s \\
& \leq 2 \delta+\int_{[a, t)_{\mathbb{T}}} L(\|x(s)-y(s)\|+\|x(\sigma(s))-y(\sigma(s))\|) \Delta s \\
& +\int_{[a, \sigma(t))_{\mathbb{T}}} L(\|x(s)-y(s)\|+\|x(\sigma(s))-y(\sigma(s))\|) \Delta s \\
& =2 \delta+\int_{a}^{t} L(\|x(s)-y(s)\|+\|x(\sigma(s))-y(\sigma(s))\|) \Delta s \\
& +\int_{a}^{\sigma(t)} L(\|x(s)-y(s)\|+\|x(\sigma(s))-y(\sigma(s))\|) \Delta s \\
& =2 \delta+2 \int_{a}^{t} L(\|x(s)-y(s)\|+\|x(\sigma(s))-y(\sigma(s))\|) \Delta s \\
& +\int_{t}^{\sigma(t)} L(\|x(s)-y(s)\|+\|x(\sigma(s))-y(\sigma(s))\|) \Delta s \\
& =2 \delta+2 \int_{a}^{t} L(\|x(s)-y(s)\|+\|x(\sigma(s))-y(\sigma(s))\|) \Delta s \\
& +L(\sigma(t)-t)(\|x(t)-y(t)\|+\|x(\sigma(t))-y(\sigma(t))\|) \\
& \leq 2 \delta+2 \int_{a}^{t} L(\|x(s)-y(s)\|+\|x(\sigma(s))-y(\sigma(s))\|) \Delta s \\
& +L(b-a)(\|x(t)-y(t)\|+\|x(\sigma(t))-y(\sigma(t))\|)
\end{aligned}
$$

and then

$$
\begin{aligned}
& \|x(t)-y(t)\|+\|x(\sigma(t))-y(\sigma(t))\| \\
& \quad \leq 2 \delta M+\int_{a}^{t} 2 L M(\|x(s)-y(s)\|+\|x(\sigma(s))-y(\sigma(s))\|) \Delta s .
\end{aligned}
$$


From Lemma 2, we get

$$
\|x(t)-y(t)\|+\|x(\sigma(t))-y(\sigma(t))\| \leq 2 \delta M e_{2 L M}(t, a)
$$

for all $t \in \mathbb{T}$. Since

$$
\|x(t)-y(t)\| \leq\|x(t)-y(t)\|+\|x(\sigma(t))-y(\sigma(t))\|
$$

the proof is complete.

\subsection{Existence to Eqs. (1) and (2)}

Let $C\left(\mathbb{T}, \mathbb{R}^{n}\right)$ denote the set of all continuous functions with domain $\mathbb{T}$ and taking values on $\mathbb{R}^{n}$.

Take a constant $\beta>0$. Define the metric $d_{\beta}: C\left(\mathbb{T}, \mathbb{R}^{n}\right) \times C\left(\mathbb{T}, \mathbb{R}^{n}\right) \rightarrow[0, \infty)$ by

$$
d_{\beta}(x, y)=\sup _{t \in \mathbb{T}} \frac{\|x(t)-y(t)\|}{e_{\beta}(t, a)}
$$

and the norms $\|\cdot\|_{\beta},\|\cdot\|_{0}: C\left(\mathbb{T}, \mathbb{R}^{n}\right) \rightarrow[0, \infty)$ by

$$
\|x\|_{\beta}=\sup _{t \in \mathbb{T}} \frac{\|x(t)\|}{e_{\beta}(t, a)}
$$

and

$$
\|x\|_{0}=\sup _{t \in \mathbb{T}}\|x(t)\| .
$$

Lemma 4 [16] If $\beta>0$ is a constant then:

1. $d_{\beta}$ is a metric;

2. $\left(C\left(\mathbb{T}, \mathbb{R}^{n}\right), d_{\beta}\right)$ is a complete metric space;

3. $\|x\|_{\beta}$ is a norm and is equivalent to the sup-norm $\|x\|_{0}$;

4. $\left(C\left(\mathbb{T}, \mathbb{R}^{n}\right),\|\cdot\|_{\beta}\right)$ is a Banach space.

Consider a complete metric space $(Y, d)$ and $F: Y \rightarrow Y$. The mapping $F$ is said to be contractive if there exists a positive constant $\lambda<1$ satisfying

$$
d(F(x), F(y)) \leq \lambda d(x, y) \quad \forall x, y \in Y .
$$

If $y \in Y$, define the sequence $\left\{F^{i}(y)\right\}$ recursively by $F^{0}(y):=y$ and $F^{i+1}(y):=$ $F\left(F^{i}(y)\right)$.

Below, we have the Banach fixed-point theorem.

Theorem 4 [4] Let $(Y, d)$ be a complete metric space and let $F: Y \rightarrow Y$ be contractive. Then, $F$ has a unique fixed point $u$ and $F^{i}(y) \rightarrow u$ for each $y \in Y$. 
Theorem 5 Take a $\Delta \times \mathcal{B}^{2 n}$-measurable function $f: \mathbb{T} \times \mathbb{R}^{n} \times \mathbb{R}^{n} \rightarrow \mathbb{R}^{n}$. Consider a function $c: \mathbb{T} \rightarrow[0, \infty)$ in $L_{1}\left([a, b)_{\mathbb{T}}\right)$. Assume that there exists $N>0$ such that

$$
\|f(t, p, q)\| \leq N(\|p\|+\|q\|)+c(t)
$$

for any $p, q \in \mathbb{R}^{n}$ and $\Delta$-a.e. $t \in[a, b)_{\mathbb{T}}$. Also assume that there exists $L>0$ satisfying

$$
\left\|f\left(t, p_{1}, p_{2}\right)-f\left(t, q_{1}, q_{2}\right)\right\| \leq L\left(\left\|p_{1}-q_{1}\right\|+\left\|p_{2}-q_{2}\right\|\right)
$$

for any $t \in \mathbb{T}$, and any $p_{1}, p_{2}, q_{1}, q_{2} \in \mathbb{R}^{n}$. If

$$
L \sup _{t \in \mathbb{T}} \mu(t)<1
$$

then the Eqs. (1) and (2) have a unique solution. Furthermore, if a sequence offunctions $\left\{x_{i}\right\}$ is defined inductively by choosing any $x_{0} \in C\left(\mathbb{T}, \mathbb{R}^{n}\right)$ and setting

$$
x_{i+1}(t)=A+\int_{[a, t)_{\mathbb{T}}} f\left(s, x_{i}(s), x_{i}(\sigma(s))\right) \Delta s
$$

then the sequence $\left\{x_{i}\right\}$ converges uniformly on $\mathbb{T}$ to the unique solution $x$ of the Eqs. (1) and (2).

Proof Take a constant $\gamma>2$ such that $L\|\mu\|_{0}=1-\frac{2}{\gamma}$ and set $\beta=L \gamma$. Let $X$ be the complete metric space $\left(C\left(\mathbb{T}, \mathbb{R}^{n}\right), d_{\beta}\right)$. Define $F: X \rightarrow X$ by

$$
(F x)(t)=A+\int_{[a, t)_{\mathbb{T}}} f(s, x(s), x(\sigma(s))) \Delta s, t \in \mathbb{T} .
$$

Let $u, v \in X$. It follows from the proof of [9, Theorem 4.3.], that

$$
\begin{aligned}
& \sup _{t \in \mathbb{T}}\left[\frac{1}{e_{\beta}(t, a)} \int_{a}^{t} L(\|u(s)-v(s)\|+\|u(\sigma(s))-v(\sigma(s))\|) \Delta s\right] \\
& \leq d_{\beta}(u, v)\left(\frac{2}{\gamma}+L\|\mu\|_{0}\right)\left[1-\frac{1}{e_{\beta}(b, a)}\right] \\
& \quad=d_{\beta}(u, v)\left[1-\frac{1}{e_{\beta}(b, a)}\right] .
\end{aligned}
$$


Hence,

$$
\begin{aligned}
& d_{\beta}(F(u), F(v)) \\
& \leq \sup _{t \in \mathbb{T}}\left[\frac{1}{e_{\beta}(t, a)} \int_{[a, t)_{\mathbb{T}}}\|f(s, u(s), u(\sigma(s)))-f(s, v(s), v(\sigma(s)))\| \Delta s\right] \\
& \leq \sup _{t \in \mathbb{T}}\left[\frac{1}{e_{\beta}(t, a)} \int_{[a, t)_{\mathbb{T}}} L(\|u(s)-v(s)\|+\|u(\sigma(s))-v(\sigma(s))\|) \Delta s\right] \\
& =\sup _{t \in \mathbb{T}}\left[\frac{1}{e_{\beta}(t, a)} \int_{a}^{t} L(\|u(s)-v(s)\|+\|u(\sigma(s))-v(\sigma(s))\|) \Delta s\right] \\
& \leq d_{\beta}(u, v)\left[1-\frac{1}{e_{\beta}(b, a)}\right] .
\end{aligned}
$$

Hence, $F$ is a contraction mapping. Using Theorem 4, we obtain the results of Theorem 5 .

Below, we state Schäfer's fixed-point theorem.

Theorem 6 [14] Let $X$ be a Banach space, $F: X \rightarrow X$ continuous and compact. Assume further that the set

$$
\Gamma=\{x \in X: x=\lambda F(x) \text { for some } \lambda \in[0,1]\}
$$

is bounded. Then, $F$ has a fixed point.

Let $X, Y$ be Banach spaces, and $C \subset X$. Mapping $F: C \rightarrow Y$ is compact if it transforms bounded sets into relatively compact sets.

In the next theorem, we obtain at least one solution to Eqs. (1) and (2).

Let $c: \mathbb{T} \rightarrow \mathbb{R}$ be a function in $L_{1}\left([a, b)_{\mathbb{T}}\right)$. We represent the integral $\int_{[a, b)_{\mathbb{T}}}|c(s)| \Delta s$ by $\|c\|_{L_{1}}$.

Theorem 7 Let $f: \mathbb{T} \times \mathbb{R}^{n} \times \mathbb{R}^{n} \rightarrow \mathbb{R}^{n}$ be a $\Delta \times \mathcal{B}^{2 n}$-measurable function and $c: \mathbb{T} \rightarrow[0, \infty)$ a function in $L_{1}\left([a, b)_{\mathbb{T}}\right)$. Suppose that for each $t \in \mathbb{T}$, the function $(p, q) \mapsto f(t, p, q)$ is continuous. Let $N>0$ be such that

$$
\|f(t, p, q)\| \leq N(\|p\|+\|q\|)+c(t)
$$

for any $p, q \in \mathbb{R}^{n}$ and $\Delta$-a.e. $t \in[a, b)_{\mathbb{T}}$. Also suppose that $N(b-a)<1$. Then, the Eqs. (1) and (2) have at least one solution.

Proof Let $X$ denote the Banach space $\left(C\left(\mathbb{T}, \mathbb{R}^{n}\right),\|\cdot\|_{0}\right)$.

Define the mapping $F: X \rightarrow X$ by

$$
(F x)(t)=A+\int_{[a, t)_{\mathbb{T}}} f(s, x(s), x(\sigma(s))) \Delta s, t \in \mathbb{T} .
$$


Consider a bounded sequence $\left\{x_{i}\right\}$ in $X$. Suppose that $\left\|x_{i}\right\|_{0} \leq \alpha$ for some $\alpha>0$. Then, the sequence $\left\{F\left(x_{i}\right)\right\}$ is bounded, since

$$
\begin{aligned}
\left\|F x_{i}\right\|_{0} & \leq\|A\|+\sup _{t \in \mathbb{T}} \int_{[a, t)_{\mathbb{T}}}\left\|f\left(s, x_{i}(s), x_{i}(\sigma(s))\right)\right\| \Delta s \\
& \leq\|A\|+\sup _{t \in \mathbb{T}} \int_{[a, t)_{\mathbb{T}}}\left(N\left\|x_{i}(s)\right\|+N\left\|x_{i}(\sigma(s))\right\|+c(s)\right) \Delta s \\
& \leq\|A\|+2 N\left\|x_{i}\right\|_{0}(b-a)+\|c\|_{L_{1}} \\
& \leq\|A\|+2 N \alpha(b-a)+\|c\|_{L_{1}} .
\end{aligned}
$$

If $t_{1}, t_{2} \in \mathbb{T}$ are such that $t_{1} \leq t_{2}$, then

$$
\begin{aligned}
\left\|\left(F x_{i}\right)\left(t_{1}\right)-\left(F x_{i}\right)\left(t_{2}\right)\right\| & \leq \int_{\left[t_{1}, t_{2}\right)_{\mathbb{T}}}\left\|f\left(s, x_{i}(s), x_{i}(\sigma(s))\right)\right\| \Delta s \\
& \leq \int_{\left[t_{1}, t_{2}\right)_{\mathbb{T}}}\left(N\left\|x_{i}(s)\right\|+N\left\|x_{i}(\sigma(s))\right\|+c(s)\right) \Delta s \\
& \leq 2 N\left\|x_{i}\right\|_{0}\left(t_{2}-t_{1}\right)+\int_{\left[t_{1}, t_{2}\right)_{\mathbb{T}}} c(s) \Delta s \\
& \leq 2 N \alpha\left(t_{2}-t_{1}\right)+\int_{\left[t_{1}, t_{2}\right)_{\mathbb{T}}} c(s) \Delta s .
\end{aligned}
$$

Hence, the sequence $\left\{F\left(x_{i}\right)\right\}$ is equicontinuous. From Arzela-Ascoli's Theorem [11], the sequence $\left\{F\left(x_{i}\right)\right\}$ has a convergent subsequence. Therefore, $F$ is compact.

Let $\bar{x} \in X$ be arbitrarily fixed. Take a sequence $\left\{x_{j}\right\}$ in $X$ obeying

$$
\lim _{j \rightarrow \infty}\left\|x_{j}-\bar{x}\right\|_{0}=0
$$

Fix a constant $\beta>0$ such that $\|\bar{x}\|_{0} \leq \beta$ and $\left\|x_{j}\right\|_{0} \leq \beta$ for all $j$. For each $t \in \mathbb{T}$,

$$
\lim _{j \rightarrow \infty}\left\|f\left(t, x_{j}(t), x_{j}(\sigma(t))\right)-f(t, \bar{x}(t), \bar{x}(\sigma(t)))\right\|=0
$$

and for $\Delta$-a.e. $t \in[a, b)_{\mathbb{T}}$,

$$
\left\|f\left(t, x_{j}(t), x_{j}(\sigma(t))\right)-f(t, \bar{x}(t), \bar{x}(\sigma(t)))\right\| \leq 4 N \beta+2 c(t)
$$

for all $j$. It follows from the dominated convergence theorem [11] that

$$
\lim _{j \rightarrow \infty} \int_{[a, b)_{\mathbb{T}}}\left\|f\left(s, x_{j}(s), x_{j}(\sigma(s))\right)-f(s, \bar{x}(s), \bar{x}(\sigma(s)))\right\| \Delta s=0
$$

and, therefore,

$$
\lim _{j \rightarrow \infty}\left\|F x_{j}-F \bar{x}\right\|_{0}=0 .
$$


Hence, $F$ is continuous in $\bar{x}$. Thus, $F$ is a continuous mapping.

Consider the set $\Gamma \subset X$ given by

$$
\Gamma=\{x \in X: x=\lambda F x \text { for some } \lambda \in[0,1]\} .
$$

If $x \in \Gamma$ it follows that

$$
\begin{aligned}
\|x(t)\|+\|x(\sigma(t))\|= & \|(\lambda F x)(t)\|+\|(\lambda F x)(\sigma(t))\| \\
\leq & 2\|A\|+\int_{[a, t)_{\mathbb{T}}}\|f(s, x(s), x(\sigma(s)))\| \Delta s \\
& +\int_{[a, \sigma(t))_{\mathbb{T}}}\|f(s, x(s), x(\sigma(s)))\| \Delta s \\
\leq & 2\|A\|+\int_{[a, t)_{\mathbb{T}}}(N\|x(s)\|+N\|x(\sigma(s))\|+c(s)) \Delta s \\
& +\int_{[a, \sigma(t))_{\mathbb{T}}}(N\|x(s)\|+N\|x(\sigma(s))\|+c(s)) \Delta s \\
\leq & 2\|A\|+\int_{a}^{t} N(\|x(s)\|+\|x(\sigma(s))\|) \Delta s \\
& +\int_{a}^{\sigma(t)} N(\|x(s)\|+\|x(\sigma(s))\|) \Delta s+2\|c\|_{L_{1}} \\
= & 2\|A\|+2 \int_{a}^{t} N(\|x(s)\|+\|x(\sigma(s))\|) \Delta s \\
& +(\sigma(t)-t) N(\|x(t)\|+\|x(\sigma(t))\|)+2\|c\|_{L_{1}} \\
\leq & 2\|A\|+2\|c\|_{L_{1}}+(b-a) N(\|x(t)\|+\|x(\sigma(t))\|) \\
& +2 \int_{a}^{t} N(\|x(s)\|+\|x(\sigma(s))\|) \Delta s
\end{aligned}
$$

and thus

$$
\begin{aligned}
\|x(t)\|+\|x(\sigma(t))\| \leq & 2\|A\| M+2 M\|c\|_{L_{1}} \\
& +\int_{a}^{t} 2 N M(\|x(s)\|+\|x(\sigma(s))\|) \Delta s
\end{aligned}
$$

for all $t \in \mathbb{T}$, where $M=\frac{1}{1-(b-a) N}$. It follows from Lemma 2 that

$$
\|x(t)\|+\|x(\sigma(t))\| \leq\left(2\|A\| M+2 M\|c\|_{L_{1}}\right) e_{2 N M}(t, a)
$$

for all $t \in \mathbb{T}$. Hence,

$$
\|x\|_{0} \leq\left(2\|A\| M+2 M\|c\|_{L_{1}}\right) e_{2 N M}(b, a)
$$

and, therefore, $\Gamma$ is bounded. 
From Theorem 6, there exists $x_{*} \in X$ such that $F x_{*}=x_{*}$. Using Lemma 3, we may conclude that $x_{*}$ is a solution to Eqs. (1) and (2).

\section{References}

1. Anderson, D.R., Boucherif, A.: Nonlocal initial value problem for first-order dynamic equations on time scales. Dyn. Contin. Discrete Impuls. Syst. Ser. A Math. Anal. 16 (Differential Equations and Dynamical Systems, suppl. S1), 222-226 (2009)

2. Bohner, M., Peterson, A.: Dynamic Equations on Time Scales: An Introduction with Applications. Birkhäuser Boston Inc., Boston (2001)

3. Cabada, A., Vivero, D.R.: Criterions for absolute continuity on time scales. J. Differ. Equ. Appl. 11(11), 1013-1028 (2005)

4. Caccioppoli, R.: Un teorema generale sull' esistenza di elementi uniti in una trasformazione funzionale. Atti Accad. Naz. Lincei Rend. VI. Ser. 11(11), 794-799 (1930)

5. Dai, Q., Tisdell, C.C.: Existence of solutions to first-order dynamic boundary value problems. Int. J. Differ. Equ. 1(1), 1-17 (2006)

6. Gilbert, H.: Existence theorems for first-order equations on time scales with $\Delta$-Carathéodory functions. Adv. Differ. Equ. 2010, Art. ID 650827, pp. 20 (2010)

7. Guseinov, G.S.: Integration on time scales. J. Math. Anal. Appl. 285(1), 107-127 (2003)

8. Hu, M., Wang, L.: Unique existence theorem of solution of almost periodic differential equations on time scales. Discret. Dyn. Nat. Soc. 2012, Art. ID 240735, pp. 8 (2012)

9. Ma, Y., Zhang, Y., Sun, J.: On qualitative analysis of delay systems and $x^{\Delta}=f\left(t, x, x^{\sigma}\right)$ on time scales. Proc. Indian Acad. Sci. Math. Sci. 120(2), 249-258 (2010)

10. Peterson, A.C., Tisdell, C.C.: Boundedness and uniqueness of solutions to dynamic equations on time scales. J. Differ. Equ. Appl. 10(13-15), 1295-1306 (2004)

11. Rudin, W.: Real and Complex Analysis, 3rd edn. McGraw-Hill Book Co., New York (1987)

12. Santos, I.L.D., Silva, G.N.: Absolute continuity and existence of solutions to dynamic inclusions in time scales. Math. Ann. 356(1), 373-399 (2013)

13. Santos, I.L.D., Silva, G.N.: Filippov's selection theorem and the existence of solutions for optimal control problems in time scales. Comput. Appl. Math. 33(1), 223-241 (2014)

14. Schaefer, H.: Über die Methode der a priori-Schranken. Math. Ann. 129, 415-416 (1955)

15. Sun, J.P., Li, W.T.: Existence of solutions to nonlinear first-order PBVPs on time scales. Nonlinear Anal. 67(3), 883-888 (2007)

16. Tisdell, C.C., Zaidi, A.: Basic qualitative and quantitative results for solutions to nonlinear, dynamic equations on time scales with an application to economic modelling. Nonlinear Anal. 68(11), 35043524 (2008)

17. Zaidi, A.H.: Existence of solutions and convergence results for dynamic initial value problems using lower and upper solutions. Electron. J. Differ. Equ. 2009, Paper no. 161, pp. 13 (2009) 